\title{
The Design of Infrared Mirror Coatings for the Enhanced Performance of Incandescent Lighting
}

\author{
Mohsen Rostami, Nima Talebzadeh, Paul G. O’Brien \\ Department of Mechanical Engineering \\ Lassonde School of Engineering, York University \\ Toronto, Canada \\ msnros@yorku.ca, paul.obrien@lassonde.yorku.ca
}

\begin{abstract}
Herein we present the design of infrared mirror coatings for the enhanced performance of incandescent lighting. We consider single and stacked dielectric mirrors comprised of alternating layers of TCO and $\mathrm{SiO}_{2}$ nanoparticle films to function as infrared mirrors that reduce heat losses in incandescent lights. In this work, thin-film theory was employed to develop MATLAB code that calculates the reflectance and transmittance spectra of dielectric mirrors. In order to validate the MATLAB code, we compared our results to experimental results reported in the literature as well as results calculated using COMSOL Multiphysics software. Our results show that an infrared dielectric mirror coated onto the glass bulb of an incandescent light can increase its efficiency by $\sim 32 \%$. However, stacked dielectric mirror coatings prevent a significant portion of visible light from transmitting through the glass bulb, and consequently decrease the efficiency of incandescent lights by $\sim 46 \%$.
\end{abstract}

Keywords- Infrared Reflectors; Thin-films; Nanoparticle Films; Lighting Technology; Optical films

\section{INTRODUCTION}

Since the invention of early commercial light bulbs in 1880 by Thomas Edison ${ }^{1}$, they have been used widely as a main source of illumination. By heating a filament wire covered by a glass bulb, the incandescent light bulb produces light. Usually the glass bulb is occupied by an inert gas to prevent the filament from burning. ${ }^{2}$ However, incandescent lights are being replaced by a new generation of more efficient lights, such as light-emitting diodes. ${ }^{3}$ One way to improve the efficiency of incandescent light bulbs is to reduce their radiative heat losses by preventing them from emitting near-infrared light. Herein we consider the design of an optical coating that achieves high reflectance in the near-infrared region and high transmittance in visible region. This can be achieved by using either a dielectric/metal film stack, ${ }^{4,5}$ a dielectric multi-layered film, ${ }^{6}$ or metallic photonic band gap (PBG) structures ${ }^{7}$. In this study, we focus on a dielectric mirror (often referred to as a Braggreflector or one-dimensional photonic crystal) comprised of alternating layers of transparent conducting oxide (TCO) and $\mathrm{SiO}_{2}$ nanoparticle (NP) films with indices of refraction of $\mathrm{n}=2$ and $n=1.3$, respectively. In previous work dielectric mirrors comprised of alternating layers of TCO and $\mathrm{SiO}_{2}$ NP films have been designed to selectively reflect light strongly over one spectral region while being highly transmissive over other spectral regions. These dielectric mirrors have been used as semi-transparent reflectors in "see-through" and multi-junction silicon-based photovoltaic cells. ${ }^{8,9}$ Furthermore, in order to expand the width of the spectral region over which the dielectric mirror reflects light, one can fabricate a coating comprised of two stacked dielectric mirrors. ${ }^{10}$ In this work we calculate and compare the efficiency of incandescent mirrors for the case in which the glass bulb is (1) bare, (2) coated with a dielectric mirror with a peak reflection in the infrared region and (3) coated with stacked dielectric mirrors that exhibit an extended reflection peak in the infrared region.

\section{METHOD}

A dielectric mirror is comprised of alternating films with a higher (denoted as $\mathrm{H}$ ) and lower (denoted as L) index of refraction. Thin-film theory can be used to calculate the infrared reflectance and visible light transmittance for a dielectric mirror ${ }^{11}$ using the refractive indices and layer thickness of the films within the dielectric stack as input. The theory uses matching boundary conditions for Maxwell's equations, and more information is available in the literature ${ }^{12}$. Using thin-film theory, and with reference to Figure 1, the reflectance, $\mathrm{R}$, and transmittance, $\mathrm{T}$, at a surface are provided by Equations 1 and 2, respectively.

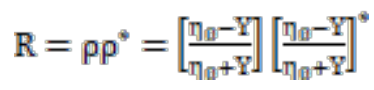

$$
\begin{aligned}
& T=\frac{4 \eta_{0} \operatorname{Re}[Y]}{\left(\eta_{0}+Y\right)\left(\eta_{0}+Y\right)^{\circ}}
\end{aligned}
$$

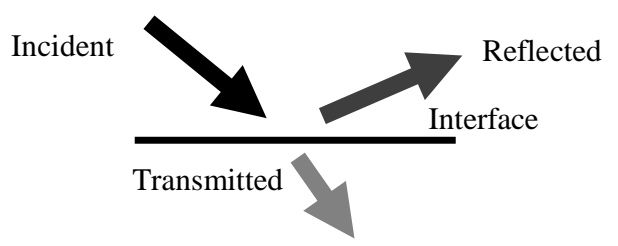

Figure 1. Transmittance and Reflection at an interface between two media 
Where $\rho$ is the amplitude of the reflection coefficient, $\eta_{0}$ represents the admittance of the incident medium and $\mathrm{Y}$ is the admittance of the medium. Also, $R e$ denotes the real part of $\mathrm{Y}$ in the Equation 2. However, Equations 1 and 2 are valid only for normal incidence. For the case of oblique incidence, one can define p-polarized and s-polarized light which are electric vectors parallel and perpendicular to the plane of incidence, respectively (Figure 2). Consequently, the new expressions will be defined with the tilted admittance for s-polarized and ppolarized light as in Equations 3 and 4:

$$
\begin{array}{ll}
\eta_{s}=y \cos \theta=(n-i k) \cos \theta & \text { for s-polarized light } \\
\eta_{p}=y / \cos \theta=(n-i k) / \cos \theta & \text { for p-polarized light }
\end{array}
$$

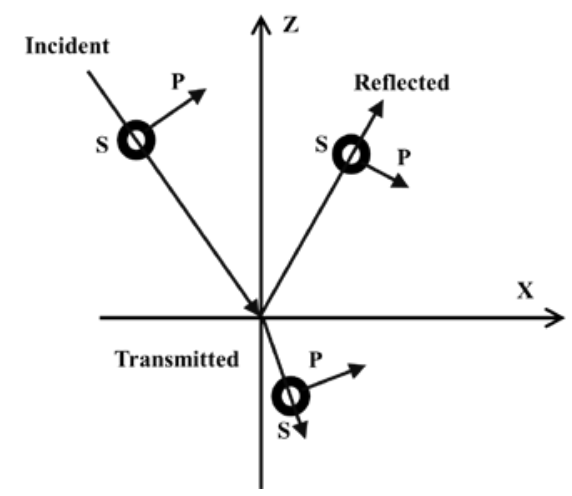

Figure 2. P-polarized and s-polarized light based for oblique incidence.

\section{A. Matlab Code Validation}

We developed a MATLAB code, based on the methods described in the previous section, that calculates the reflectance and transmittance spectra of dielectric mirrors. To validate the code we calculated and compared the reflectance and transmittance of dielectric mirrors to those studied in the literature. For example, we calculated the reflectance from a dielectric mirror comprised of nine layers of polystyrene and tellurium and the results are compared to experimentally measured results ${ }^{12}$ in Figure 3. We also calculated the transmittance of dielectric mirrors comprised of alternating layers of indium-tin oxide (ITO) and $\mathrm{SiO}_{2}$ nanoparticle films and compared our results to experimentally measured results ${ }^{10}$. As shown in Figure 4, the reflectance and transmittance spectra calculated using our MATLAB code provide a good approximation to those reported in the literature. Discrepancies between the calculated and experimentally measured spectra shown in Figures 3 and 4 are attributed to differences between the thickness or index of refraction values used in the experiment as compared to the values assigned to parameters in the analytical method. We also compared reflectance and transmittance spectra calculated using our MATLAB code and COMSOL Multiphysics software (version 5.3), which offers two separate modules that can be used for thin-film calculations (Ray Optics and Wave Optics). The results from our MATLAB code are compared with those from COMSOL in Figures 5 and 6 . The results from both ray optics and wave optics modules agree well with those of the MATLAB code.

\section{DIELECTRIC MIRROR DESIGN}

We design a one-dimensional dielectric mirror (otherwise referred to as a Bragg-reflector) to function as a coating for an incandescent light bulb that internally reflects infrared radiation back towards the light filament to reduce radiative heat losses. The term "one-dimensional" in our case refers to the fact that there is no variation in the dielectric function, $\varepsilon$, along directions excluding (z). ${ }^{14}$ In this report, TCO $(n=2)$ is assumed to be the high index of refraction film, represented by $\mathrm{H}$, and a $\mathrm{SiO}_{2}$ NP film $(\mathrm{n}=1.3)$ is assumed to be the low index of refraction film, which is represented as L in the stacking sequence shown in Figure 7.

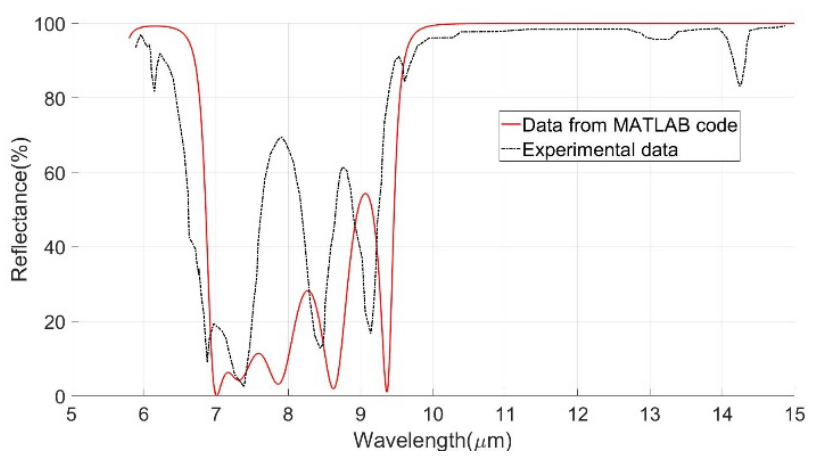

Figure 3. Experimentally measured and calculated (MATLAB code) reflectance spectra of a dielectric mirror comprised of nine layers of polystyrene and tellurium ${ }^{11}$.

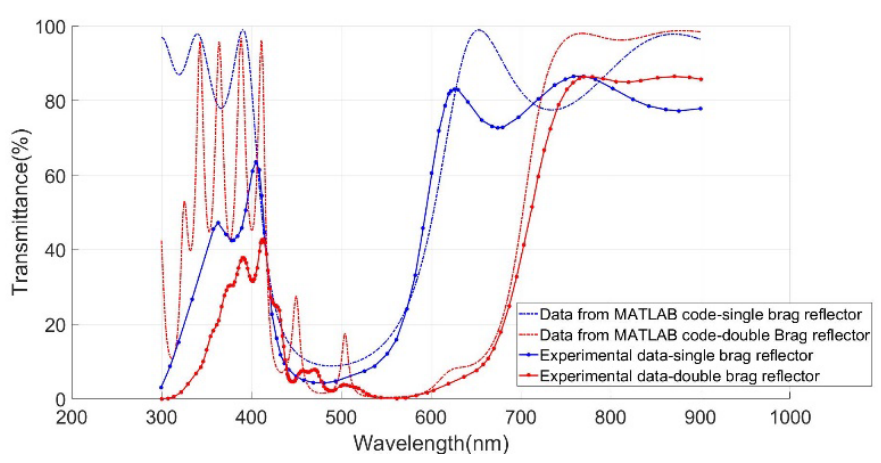

Figure 4. Experimentally measured and calculated (MATLAB code) transmittance spectra of dielectric mirrors comprised of alternating layers of ITO and $\mathrm{SiO}_{2}$ nanoparticle films ${ }^{8}$.

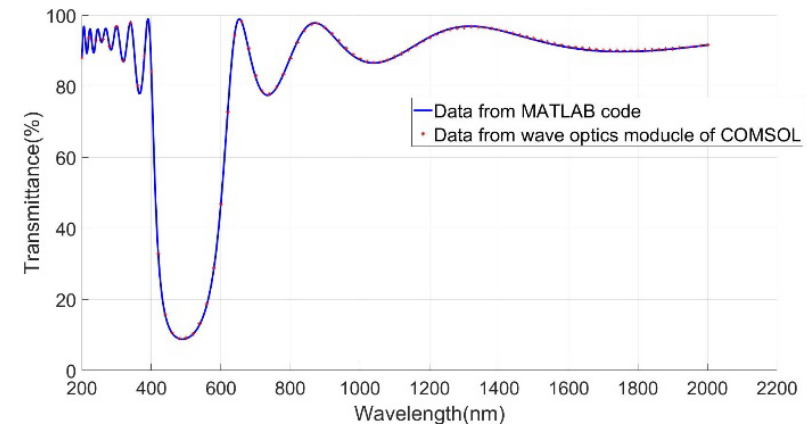

Figure 5. Transmittance of a dielectric mirror comprised of alternating layers of TCO and $\mathrm{SiO}_{2}$ nanoparticle films calculated using our MATLAB code and

COMSOL Multiphysics software (five layers of TCO and $\mathrm{SiO}_{2}$ with thicknesses of 60 and $90 \mathrm{~nm}$, respectively ). 
Figure 7 shows a dielectric mirror with a stacking sequence of $[\mathrm{HLH}]^{10}$ where $\mathrm{H}$ and $\mathrm{L}$ represent the TCO and $\mathrm{SiO}_{2} \mathrm{NP}$ films, respectively. As discussed in the following section, the thicknesses of the TCO and $\mathrm{SiO}_{2}$ films were chosen such that the spectral position of the reflectance peak minimizes the radiative heat losses from the incandescent bulb.

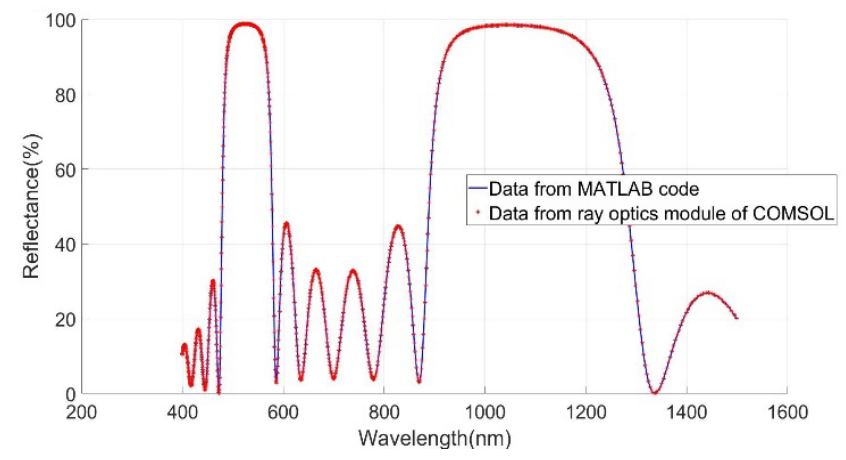

Figure 6. Reflectance spectra from a dielectric mirror comprised of alternating layers of TCO and $\mathrm{SiO}_{2}$ nanoparticle films calculated using our MATLAB code and COMSOL Multiphysics software (five layers of TCO and

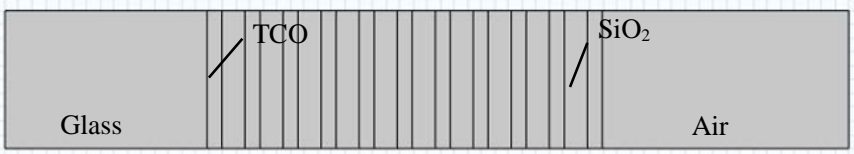

$\mathrm{SiO}_{2}$ with thicknesses of 73 and $257 \mathrm{~nm}$, respectively ).

Figure 7. A one-dimensional dielectric mirror comprised of alternating layers of TCO and $\mathrm{SiO}_{2}$ films. This dielectric mirror is coated onto glass.

\section{A. Light Bulb Blackbody Radiation Spectrum}

Herein we assume the spectral distribution of radiation emitted from an incandescent bulb approximates that of a blackbody at a temperature of $3300 \mathrm{~K}$, which is the operating temperature of an incandescent light bulb filament ${ }^{15}$. As can be seen in the Figure 8, the highest intensity emitted from a blackbody at a temperature of $3300 \mathrm{~K}$ occurs at a wavelength of $880 \mathrm{~nm}$, and therefor we choose this as the reference wavelength for the Bragg-peak position of the dielectric mirror. It follows that the thicknesses of the TCO and $\mathrm{SiO}_{2}$ nanoparticle films in a quarter-wave stack with a reflectance peak wavelength of $880 \mathrm{~nm}$ is $110 \mathrm{~nm}$ and $169.5 \mathrm{~nm}$, respectively. Considering the dielectric mirror with a stacking sequence of $[\mathrm{HLH}]^{10}$ shown in Figure 7, the values of $\mathrm{H}$ and $\mathrm{L}$ would be $55 \mathrm{~nm}$ and $169.5 \mathrm{~nm}$, respectively. The superscript ' 10 ' indicates that the [HLH] stacking sequence is repeated 10 times; in this case the uppermost and bottommost films are 55 $\mathrm{nm}$ thick TCO films, which act as optical matching layers that reduce reflection outside the Bragg-reflection peak.

\section{B. Results}

The reflectance spectrum for the $[\mathrm{HLH}]^{10}$ stack comprised of $110 \mathrm{~nm}$ thick TCO films and $169.5 \mathrm{~nm}$ thick $\mathrm{SiO}_{2} \mathrm{NP}$ films is shown in Figure 9. This reflectance spectrum exhibits a strong reflectance peak with a full-width at half-maximum of
$360 \mathrm{~nm}$, starting at $770 \mathrm{~nm}$ and ending at $1030 \mathrm{~nm}$, and a maximum reflectance value of $\sim 100 \%$ in the spectral vicinity of $880 \mathrm{~nm}$. The other spectral regions, including the visible and infrared, show some fluctuations with the reflectance value below 30\% over most of these regions. As shown in Figure 8, a large portion of the radiation emitted from a blackbody at a temperature of $3300 \mathrm{~K}$ is emitted with a wavelength greater than $1030 \mathrm{~nm}$. In order to extend the high reflectance values for the quarter wave stack shown in Figure 9 to wavelengths beyond $1030 \mathrm{~nm}$, one can stack a second dielectric mirror onto the original dielectric mirror, which will be discussed in the next section.

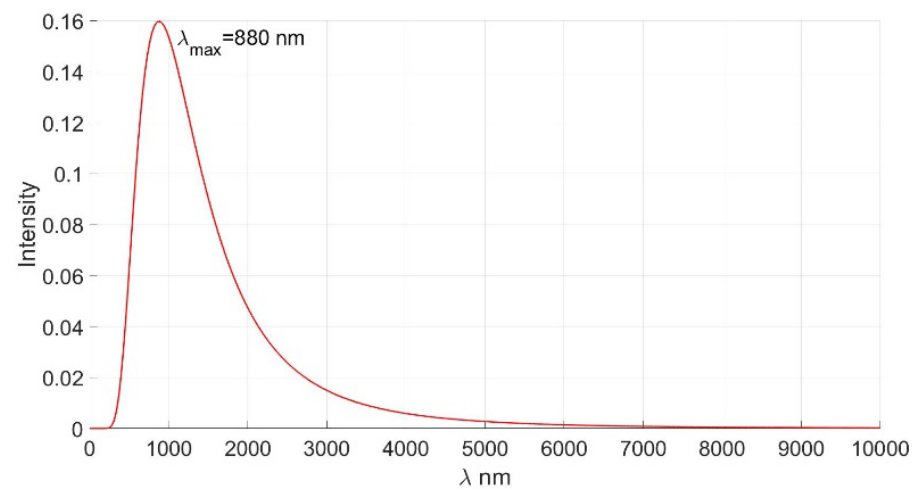

Figure 8. Radiation spectrum for a blackbody at a temperature of $3300 \mathrm{~K}$.

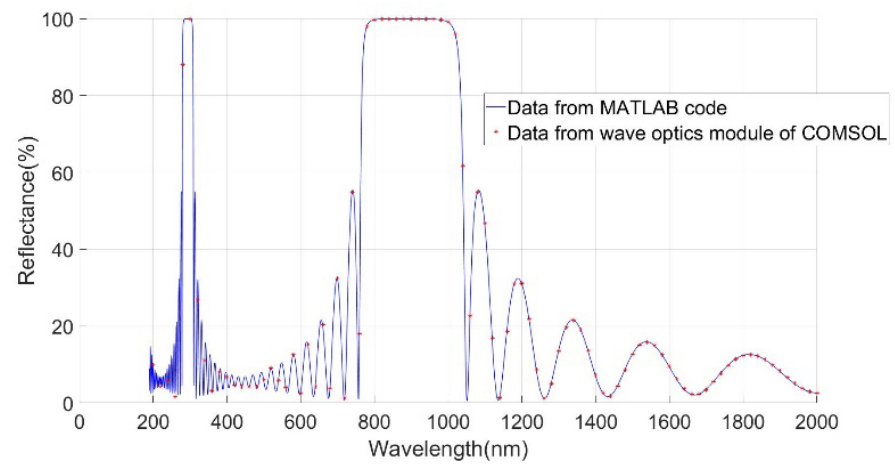

Figure 9. Reflectance spectrum for a quarter-wave stack comprised of alternating layers of TCO and $\mathrm{SiO}_{2}$ NP films with thicknesses of $110 \mathrm{~nm}$ and $169.5 \mathrm{~nm}$, respectively.

\section{DOUBlE BRAgG REFLECTOR DESIGN AND CORRESPONDING RESULTS}

The reflectance spectra shown in Figure 9 can be extended to wavelengths greater than $1030 \mathrm{~nm}$ by stacking the initial dielectric mirror, which has a peak reflection wavelength of $880 \mathrm{~nm}$, with a second dielectric mirror that has a different reflection peak position. The stacked dielectric mirrors, shown in Figure 10 and referred to as a 'double stack', can be designed with a layered configuration denoted as $[\mathrm{hLh}]^{10}[\mathrm{HLH}]^{10}$. In this notation $[\mathrm{HLH}]^{10}$ represents the initial quarter-wave stack comprising 10 bilayers with $\mathrm{L}=169.5 \mathrm{~nm}$ thick $\mathrm{SiO}_{2} \mathrm{NP}$ films and $\mathrm{H}=55 \mathrm{~nm}$ thick TCO films. The second dielectric stack, denoted as $[\mathrm{hLh}]^{10}$, is also comprised of TCO and $\mathrm{SiO}_{2}$ films, although the thickness of the TCO films 
have been increased to $169.5 \mathrm{~nm}$ (with $\mathrm{h}=84.75 \mathrm{~nm}$ ) to move the reflection peak position further into the infrared spectral region. The maximum Bragg-peak reflectance wavelength for the $[\mathrm{hLh}]^{10}$ stack is $\lambda_{\mathrm{p}}=2 .\left(\mathrm{t}_{\mathrm{L}} \cdot \mathrm{n}_{\mathrm{L}}+\mathrm{t}_{\mathrm{H}} \cdot \mathrm{n}_{\mathrm{h}}\right) \approx$ $2 \times(169.5 \mathrm{~nm} \cdot 2+169.5 \mathrm{~nm} \cdot 1.3) \approx 1119 \mathrm{~nm}$.

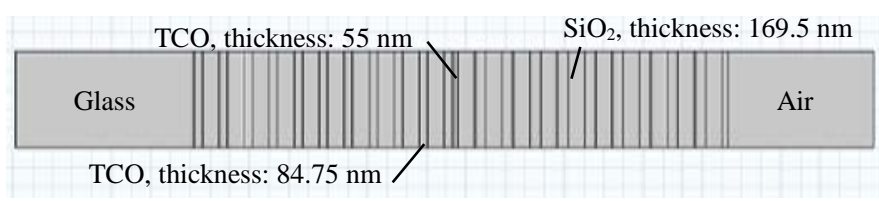

Figure 10. The one-dimensional multilayer film used for the single Bragg reflector design

The reflectance spectra calculated using our MATLAB code for the single and double-stacked dielectric mirrors are shown in Figure 11. The double-stacked dielectric mirror is strongly reflecting over a broad spectral region extending beyond 800 to $1200 \mathrm{~nm}$. We determine the amount of radiant power emitted from the incandescent light by multiplying the blackbody radiation spectra from the light filament with the transmission spectra of the glass bulb for the cases in which it is bare, coated with a single dielectric mirror and coated with the stacked dielectric mirror, and the results are shown in Figure 12.

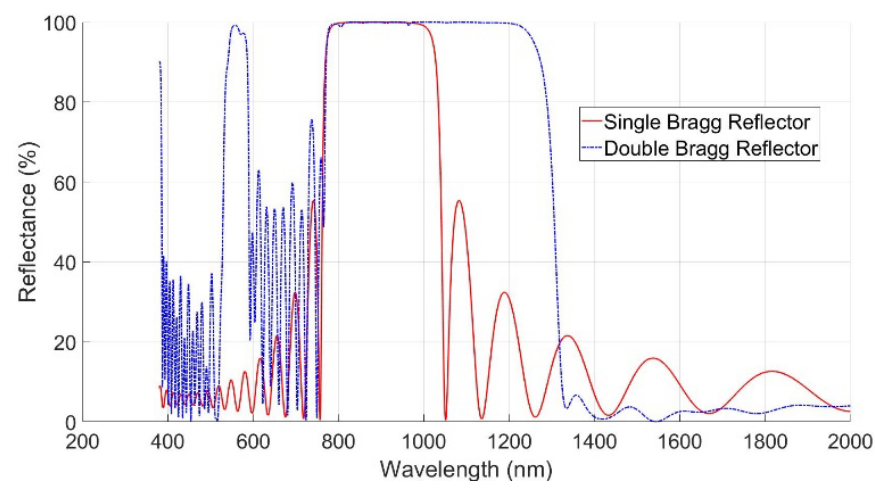

Figure 11. Reflectance spectrum for the "double stack" comprised of alternating layers of TCO and $\mathrm{SiO}_{2} \mathrm{NP}$ films with a $[\mathrm{hLh}]^{10}[\mathrm{HLH}]^{10}$ stacking sequence

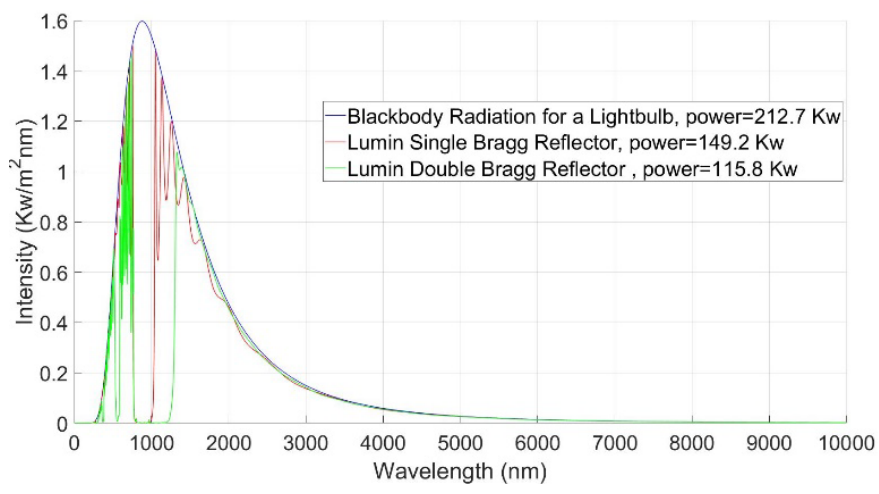

Figure 12. The radiation spectra for a blackbody at a temperature of $3300 \mathrm{~K}$ (blue curve) and the intensity of radiation emitted from a blackbody that is transmitted through the $[\mathrm{HLH}]^{10}$ Bragg reflector and the "double-stack" with a $[\mathrm{hLh}]^{10}[\mathrm{HLH}]^{10}$ stacking sequence.

\section{LIGHT BULB EFFICIENCY}

The lumens emitted from a light bulb with a filament at a temperature of $3300 \mathrm{~K}$ for the case in which the glass bulb is: bare, coated with the $[\mathrm{HLH}]^{10}$ quarter wave stack, or coated with the "double-stack" with a $[\mathrm{hLh}]^{10}[\mathrm{HLH}]^{10}$ stacking sequence, are shown in Figure 13. For each of these cases, the transmitted irradiance of the light bulb was multiplied by the CIE 1978 eye sensitivity function ${ }^{17}$ to determine the transmitted lumens ${ }^{18}$. The lumens transmitted for the case of the stacked dielectric mirror is a lot less than the lumens transmitted for the case of the bare glass bulb or single dielectric mirror coating. The corresponding values are equal to 9.51, 8.785 and $3.44 \mathrm{Klm} / \mathrm{m}^{2}$ for the case the bare lightbulb, the single dielectric mirror and the stacked mirror, respectively.

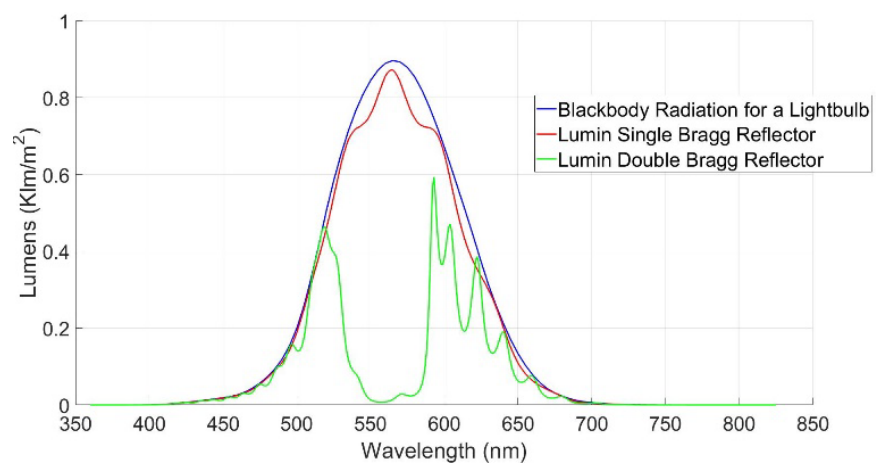

Figure 13. The lumens emitted from a light bulb with a filament at a tempeature of $3300 \mathrm{~K}$ for the case in which the glass bulb is: bare (blue curve), coated with the $[\mathrm{HLH}]^{10}$ quarter wave stack (red curve), or coated with the "double-stack" with a $[\mathrm{hLh}]^{10}[\mathrm{HLH}]^{10}$ stacking sequence.

In this work we assume the temperature of the filament in the incandescent light is $3300 \mathrm{~K}$, regardless of what coating is applied to the bulb. However, the power consumed by the filament to reach a temperature of $3300 \mathrm{~K}$ depends on the optical coating applied to the glass bulb. The efficiency of the bulb can be estimated using Equation 5.

$$
\eta=\frac{\Phi}{\text { power }}
$$

Where the power in the denominator is the total radiant power emitted by the light over all spectral regions, and $\Phi$ is the power of light emitted in the visible spectral region. It follows that the efficiency of the incandescent light bulb for the case in which the glass bulb is bare is $4.47 \%$. Moreover, this efficiency can be increased to $5.89 \%$ by coating the glass bulb with the dielectric mirror comprised of TCO and $\mathrm{SiO}_{2} \mathrm{NP}$ films with a $[\mathrm{HLH}]^{10}$ stacking sequence and a Bragg-reflectance peak position of $880 \mathrm{~nm}$. However, the efficiency decreases to $2.97 \%$ if the glass bulb is coated with the "double-stack" with the $[\mathrm{hLh}]^{10}[\mathrm{HLH}]^{10}$ stacking sequence.

\section{CONCLUSION}

In this work we presented the design of a dielectric mirror coating that improves the efficiency of incandescent light bulbs. The dielectric mirror is comprised of alternating layers of TCO and $\mathrm{SiO}_{2} \mathrm{NP}$ films with indices of refraction of 2 and 1.3, respectively. The thicknesses of these $\mathrm{TCO}$ and $\mathrm{SiO}_{2}$ are 
set such that the dielectric mirror has a peak reflectance value at the same wavelength as the maximum of the radiation spectrum of the filament within the incandescent bulb. For example, we considered a filament operating temperature of $3300 \mathrm{~K}$, which has a radiation spectrum peak value of $880 \mathrm{~nm}$, and the dielectric mirror was designed such that its peak reflectance value is also at $880 \mathrm{~nm}$. Our results show that the dielectric mirror coating increases the efficiency of the incandescent light by $32 \%$.

\section{ACKNOWLEDGEMENTS}

This research was supported by the Natural Sciences and Engineering Research Council of Canada

\section{REFERENCES}

[1] F. Robert, and P. Israel, Edison's electric light: biography of an invention. New Brunswick, New Jersey: Rutgers University Press, 1986

[2] M. K. Giridharan, Electrical Systems Design, Chapter 3, 2010

[3] O. Ilic et all, Tailoring high-temperature radiation and the resurrection of the incandescent source, Nature Nano Technology, 2016

[4] J. Brett et all, Development of high energy-conserving incandescent lamps, J. Illuminating Eng. Soc. 214, p. 93, 1980

[5] R. Fontanta et all, The design, construction and performance of an incandescent light source with a transparent heat mirror, Lighting Tech. 18, p. 93, 1986.
[6] Y. S. Kim et all, Analysis of photon recycling using metallic photonic crystal, J. Appl. Phys. 102, p. 063107, 2007.

[7] Shawn-Yu Lin and Yong-Sung Kim, A cool light bulb, SPIE, 2008

[8] Y. Yang, P. G. O’Brien, G. A. Ozin, N. P. Kherani, "See-through amorphous silicon solar cells with selectively transparent and conducting photonic crystal back reflectors for building integrated photovoltaics" Applied Physics Letters 103 (22), 221109

[9] B. Ramautarsingh, P O'Brien, A. Flood, N. Kherani, “Quantum efficiency enhancement in multi-junction solar cells with spectrally selective and conducting 1D photonic crystals” Journal of Materials Chemistry C, 2016, 4, 9276-9286

[10] P. G. O’Brien et all, Selectively transparent and conducting photonic crystal solar spectrum splitters made of alternating sputtered indium-tin oxide and spin-coated silica nanoparticle layers for enhanced photovoltaics, Solar Energy Materials and Solar Cells, pp. 173-183, 2012

[11] J. Zhang, Hyper-NA optical systems and applications in sample measurement, Appendix F, Thesis Report, University of Arizona, 2010

[12] H. A. Macleod, Thin Films Optical Filters, Fourth Edittion, Taylor and Francis Group, 2010

[13] Y. Fink, A Dielectric Omnidirectional Reflector, Science, Vol. 282, 1998

[14] J. D. Joannopoulos et all, Photonic Crystals: Molding the Flow of Light, Princeton University Press, Chapter 4 - The Multilayer Film: A OneDimensional Photonic Crystal, 2008

[15] A. C. Wilbraham et all, Prentice Hall Chemistry, Pearson Prentice Hall, 2006

[16] G. B. Rybicki, Alan P. Lightman, Radiative Processes in Astrophysics, John Wiley \& Sons, 1979

[17] E. F. Schubert, Light Emitting Diodes, Second edition, Chapter 16, 2006

[18] P. G. O’Brien et all, Selectively transparent and conducting photonic crystal rear-contacts for thin-film silicon-based building integrated photovoltaics, Opt. Exp., Vol. 19, No. 18, 2011 